\title{
Nonparaxial wave beams in a uniaxial birefringent crystal
}

\author{
Fadeyeva T.
}

Physics Department, Taurida National V. I. Vernadsky University, 4 Vernadsky Ave., 95007 Simferopol, Ukraine, e-mail: tatyana.fadeyeva@gmail.com

Received: 05.11.2009

\begin{abstract}
The solutions are obtained to vector wave equation for nonparaxial beams propagating along the optic axis in a uniaxial birefringent crystal. We have revealed that circularly polarised nonparaxial beams may be presented as a set of transverse electric and transverse magnetic waves keeping their structure up to a scale factor when propagating. A beam with arbitrary field distribution may be written as a composition of such the wave fields. We have shown that a circularly symmetric vortex beam with the initial circular polarisation preserves its structure inside the crystal. A circular symmetry of nonparaxial vortex beam with the initial linear polarisation gets lost as the beam transmits through the crystal. The circular symmetry is not recovered in the paraxial case, being an inherent property of the linearly polarised beams.
\end{abstract}

Keywords: nonparaxial beam, optical vortex, uniaxial birefringent crystal

PACS: 42.15.-i, 42.81.Gs

UDC: $535.3,535.5$

\section{Introduction}

Propagation of vortex beams in anisotropic crystals is of obvious interest from both practical and theoretical points of view. On the one hand, modern technology needs special tools permitting to trap and transport micro objects. Optical vortices embedded in light beams can implement this task. A comparatively simple way to produce singular beams is a computer-generated hologram technique (or phase masks method) [1]. However, it is very difficult to employ the holograms in real-time-scale systems without special complex mechanical gadgets. At the same time, optically uniaxial crystals are capable of performing the same operations without additional devices [2, 3].

On the other hand, theoretical description of singular optical processes in optically birefringent crystals encounters too often mathematical difficulties. In particular, solutions to the Maxwell equations involve evolution integrals that very seldom lead to closed expressions. As a rule, one obtains uncertain results for tightly focused (i.e., nonparaxial) beams, which are right out of a major interest for modern technologies.

To the present time, there has been a great number of works targeting propagation of nonparaxial beams in free space or homogeneous media (see, e.g., [4-10] and references therein). The key point in these studies is a right choice of vector-potential that can lead 
to a wanted solution. A classical way for analysing the propagation of light in crystals is a plane-wave technique [11] that enables one to probe each direction in a crystal, looking after the polarisation state transformations. However, any beam involves a great number of rays (plane waves) propagating in different directions with different velocities. In order to reproduce a picture as a whole, it is necessary to use a spectral integral technique [5]. Employing of this method for paraxial beams propagating through both uniaxial [12-14] and biaxial [15-17] crystals has entailed fruitful results, while the expansion of this technique to nonparaxial beams again has resulted in solutions that need asymptotic or numerical methods for their analysis.

Another approach to the problem is a technique of wave modes with eigen polarisations or, otherwise, a complex-source-point technique [18-22]. This method has been used for the first time for a tilted propagation of extraordinary Gaussian beam in a uniaxial birefringent crystal (see the work [19]). In the studies [23, 24], variations of the above method have been employed for tilted vortex beams of the highest orders.

The aim of our work is to study the main properties of nonparaxial beams which have their analogues among Laguerre-Gaussian paraxial beams propagating along the optic axis of uniaxial birefringent crystals, using the technique of wave modes with eigen polarisations.

\section{Generatrix beams}

Let us consider at first a general solution to the Maxwell equations for the waves propagating in an optically uniaxial birefringent crystal, with the permittivity tensor presented as $\hat{\varepsilon}=\operatorname{diag}\left(\varepsilon, \varepsilon, \varepsilon_{3}\right)$. For the case of monochromatic waves containing the factor $\exp \{i \omega t\}$, the Maxwell equations acquire the following form:

$$
\begin{aligned}
& \nabla \times \mathbf{E}=-i k_{0} \mathbf{H}, \nabla \times \mathbf{H}=i k_{0} \hat{\varepsilon} \mathbf{E}, \\
& \nabla \hat{\varepsilon} \mathbf{E}=0, \quad \nabla \mathbf{H}=0 .
\end{aligned}
$$

Among a variety of different vector-potential forms used for free-propagating nonparaxial beams [5], we choose the solutions for anisotropic case which possess a circular symmetry of circularly polarised components of the field. Such a requirement corresponds to two different cases related to (1) a transverse electric wave $\left(E_{z}=0\right)$ and (2) a transverse magnetic one $\left(H_{z}=0\right)$, where the vector-potential $\mathbf{A}$ in the work [8] is directed along $z$ axis.

(1) The transverse electric wave field $\left(E_{z}=0\right)$ :

The field components of the electric field may be presented as

$$
E_{x}=\partial_{y} \Psi_{1}, \quad E_{y}=-\partial_{x} \Psi_{1}, \quad E_{z}=0 .
$$

From Eq. (1) one can write

$$
(\nabla \times \mathbf{E})_{z}=-i k_{0} H_{z},
$$

so that the longitudinal component of the magnetic field is as follows: 


$$
H_{z}=-\frac{i}{k_{0}} \nabla_{\perp}^{2} \Psi_{1}
$$

Moreover, we have

$$
\nabla \mathbf{H}=\partial_{x} H_{x}+\partial_{y} H_{y}-\frac{i}{k_{0}} \partial_{z} \nabla_{\perp}^{2} \Psi_{1}=0 .
$$

Thus, the transverse components of the magnetic field may be written as

$$
H_{x}=\frac{i}{k_{0}} \partial_{x z}^{2} \Psi_{1}, \quad H_{y}=\frac{i}{k_{0}} \partial_{y z}^{2} \Psi_{1} .
$$

In order to find equation for the function $\Psi_{1}$, we use the relation

$$
(\nabla \times \mathbf{H})_{x}=-i k_{0} \varepsilon E_{x} .
$$

Substituting Eqs. (3) and (4) in the above equation, we come to the Helmholtz scalar equation:

$$
\nabla_{\perp}^{2} \Psi_{1}+\partial_{z}^{2} \Psi_{1}+k_{0}^{2} \varepsilon \Psi_{1}=0
$$

The wave of such a type spreads over a crystal in a manner similar to the case of homogeneous medium with the refractive index $n_{o}=\sqrt{\varepsilon}$. We call this wave beam as $a$ nonparaxial ordinary generatrix beam.

(2) The transverse magnetic field $\left(H_{z}=0\right)$ :

Let us choose the components of the magnetic field as

$$
H_{x}=\partial_{y} \Psi_{2}, \quad H_{y}=-\partial_{x} \Psi_{2}, \quad H_{z}=0 .
$$

Then we obtain from Eq. (1)

$$
(\nabla \times \mathbf{H})_{z}=i k_{0} \varepsilon_{3} E_{z},
$$

so that the longitudinal component of the electric field is given by

$$
E_{z}=\frac{i}{k_{0} \varepsilon_{3}} \nabla_{\perp}^{2} \Psi_{2}
$$

Further, from the condition $\nabla \hat{\varepsilon} \mathbf{E}=0$ we find

$$
\varepsilon\left(\partial_{x} E_{x}+\partial_{y} E_{y}\right)+\varepsilon_{3} \partial_{z}\left(\frac{i}{k_{0} \varepsilon_{3}} \nabla_{\perp}^{2} \Psi_{2}\right)=0
$$

so that

$$
E_{x}=-\frac{i}{k_{0} \varepsilon} \partial_{x z}^{2} \Psi_{2}, \quad E_{y}=-\frac{i}{k_{0} \varepsilon} \partial_{y z}^{2} \Psi_{2}
$$

Equation for the $\Psi_{2}$ function may be found basing on the relation

$$
(\nabla \times \mathbf{E})_{x}=-i k_{0} H_{x} .
$$

It has the following form:

$$
\nabla_{\perp}^{2} \Psi_{2}+\frac{\varepsilon_{3}}{\varepsilon} \partial_{z}^{2} \Psi_{2}+k_{0}^{2} \varepsilon_{3} \Psi_{2}=0
$$


Eq. (9) shows that the wave beam has a new scale over the $z$ axis $\left(z^{\prime}=\sqrt{\varepsilon / \varepsilon_{3}} z\right)$ and propagates with a new wave number $\left(k_{e}=k_{0} \sqrt{\varepsilon_{3}}\right)$. We call this wave beam as a nonparaxial extraordinary generatrix beam.

The other types of vector-potential results in non-symmetric solutions associated with deformed intensity distributions over the beam cross-section.

\section{Vortex beam propagating along the optic axis of crystals 3.1. Mode fields}

A particular wave solution to the Helmholtz equation (5) in the spherical coordinates may be written as [4]

$$
\bar{\Psi}_{1}=j_{n}\left(k_{o} \bar{R}\right) P_{n}^{m}(\cos \bar{\theta}) \exp (i m \varphi),
$$

where $j_{n}(x)$ stands for the spherical Bessel function of the first kind, $P_{n}^{m}(x)$ is the Legendre polynomial, $\bar{R}=\sqrt{r^{2}+z^{2}}$ and $\cos \bar{\theta}=\frac{z}{\bar{R}}$. In order to form the wave function of the beam propagating along $z$ axis, we make the transformation $z \rightarrow z+i z_{o}$, where $z_{o}$ is a characteristic parameter of the ordinary beam. The shift of a point light source to imaginary region is equivalent to violation of spherical symmetry of the wave and its deformation along the $z$ axis without breaking the axial symmetry, the solution given by Eq. (10) as before obeying the wave equation [6-9]. Then the wave function of the ordinary beam becomes

$$
\Psi_{1}^{(n, m)}=j_{n}\left(k_{o} R_{o}\right) P_{n}^{m}\left(\cos \theta_{o}\right) \exp (\operatorname{im} \varphi),
$$

with $R_{o}=\sqrt{r^{2}+\left(z+i z_{o}\right)^{2}}$ and $\cos \theta_{o}=\frac{z+i z_{o}}{R_{o}}$.

Correspondingly, a particular solution to Eq. (9) for the extraordinary beams is

$$
\Psi_{2}^{(n, m)}=j_{n}\left(k_{e} R_{e}\right) P_{n}^{m}\left(\cos \theta_{e}\right) \exp (i m \varphi),
$$

with $R_{e}=\sqrt{r^{2}+\left(z^{\prime}+i z_{e}\right)^{2}}$ and $\cos \theta_{e}=\frac{z^{\prime}+i z_{e}}{R_{e}}$, where $z_{e}$ stands for a characteristic parameter of the extraordinary beam.

We choose the wave beams of the lowest order

$$
\begin{aligned}
& \Psi_{1}^{(0,0)}=\Psi_{1}\left(x, y, z, k_{o}\right)=\frac{j_{0}\left(k_{o} R_{o}\right)}{j_{0}\left(i k_{o} z_{o}\right)}, \\
& \Psi_{2}^{(0,0)}=\Psi_{2}\left(x, y, z^{\prime}, k_{e}\right)=\frac{j_{0}\left(k_{e} R_{e}\right)}{j_{0}\left(i k_{e} z_{e}\right)}
\end{aligned}
$$

as generatrix functions, while the lowest-order spherical Bessel function is given by

$$
j_{0}(x)=\frac{\sin x}{x} \text {. }
$$


The electric field of the lowest-order ordinary vortex beam may be found from Eq. (2). However, it makes a sense to use new variables $u=x+i y, v=x-i y$ and a circularly polarised basis $E_{+}=E_{x}-i E_{y}, E_{-}=E_{x}+i E_{y}$. In this case the electric field of the ordinary beam becomes

$$
E_{+}^{o}=\partial_{u} \Psi_{1}, E_{-}^{o}=-\partial_{v} \Psi_{1}, \quad E_{z}^{o}=0,
$$

whereas the electric field of the extraordinary beam is given by the relations

$$
\begin{aligned}
& \bar{E}_{+}=-\frac{i}{k_{0} \varepsilon} \partial_{u z}^{2} \Psi_{2}, \quad \bar{E}_{-}=-\frac{i}{k_{0} \varepsilon} \partial_{v z}^{2} \Psi_{2}, \\
& \bar{E}_{z}=\frac{i}{k_{0} \varepsilon_{3}} \nabla_{\perp}^{2} \Psi_{2}=4 \frac{i}{k_{0} \varepsilon_{3}} \partial_{u v} \Psi_{2} .
\end{aligned}
$$

Now let us form new extraordinary beam fields given by Eq. (8):

$$
\begin{aligned}
& E_{1,+}^{e}=-\frac{k_{0} \varepsilon}{i} \int \bar{E}_{+} d z=\int \partial_{u z}^{2} \Psi_{2} d z=\partial_{u} \Psi_{2}, \\
& E_{1,-}^{e}=-\frac{k_{0} \varepsilon}{i} \int \bar{E}_{-} d z=\int \partial_{v z}^{2} \Psi_{2} d z=\partial_{v} \Psi_{2}, \\
& E_{z}^{e}=-4 \frac{\varepsilon}{\varepsilon_{3}} \int \partial_{u v} \Psi_{2} d z .
\end{aligned}
$$

These equations enable us to treat the ordinary and extraordinary beams in the same form. Notice that the components of the former magnetic field must be integrated, too.

\subsection{Vortex beam of the lowest order}

Our aim is to match the beam we can produce at the crystal input $(z=0)$ in an isotropic medium with the refractive index $n_{o}$ with the beam field inside a crystal (in the same $z=0$ plane). Our requirement is that the left-hand polarised (LHP) component of the beam $E_{0,-}(z=0)$ should vanish in the plane $z=0$. We also assume the reflected wave to be negligibly small. Let us first verify whether the superposition

$$
\mathbf{E}_{1}=a \mathbf{E}_{1}^{o}+b \mathbf{E}_{1}^{e}
$$

corresponds to our requirement for the transverse field components. Using Eqs. (13), (14), (16), (19) and (20), we write

$$
E_{1,+}=\partial_{u}\left(\Psi_{1}+\Psi_{2}\right), \quad E_{1,-}=-\partial_{v}\left(\Psi_{1}-\Psi_{2}\right),
$$

where $a$ and $b$ are constants. Let us choose a new normalisation in the form

$$
a=-\frac{2 i z_{o}}{k_{o} w_{0}} \frac{j_{0}\left(i k_{o} z_{o}\right)}{j_{1}\left(i k_{o} z_{o}\right)}, \quad b=-\frac{2 i z_{e}}{k_{e} w_{0}} \frac{j_{0}\left(i k_{e} z_{e}\right)}{j_{1}\left(i k_{e} z_{e}\right)}
$$

and obtain

$$
E_{1,+}=\frac{v}{w_{0}}\left[\frac{i z_{o}}{R_{o}} \frac{j_{1}\left(k_{o} R_{o}\right)}{j_{1}\left(i k_{o} z_{o}\right)}+\frac{i z_{e}}{R_{e}} \frac{j_{1}\left(k_{e} R_{e}\right)}{j_{1}\left(i k_{e} z_{e}\right)}\right],
$$




$$
E_{1,-}=-\frac{u}{w_{0}}\left[\frac{i z_{o}}{R_{o}} \frac{j_{1}\left(k_{o} R_{o}\right)}{j_{1}\left(i k_{o} z_{o}\right)}-\frac{i z_{e}}{R_{e}} \frac{j_{1}\left(k_{e} R_{e}\right)}{j_{1}\left(i k_{e} z_{e}\right)}\right],
$$

with $w_{0}$ being some constant a physical meaning of which will be defined later on. Besides, we made use of the relation

$$
\left(\frac{d}{x d x}\right)^{m}\left(\frac{j_{n}(x)}{x^{n}}\right)=(-1)^{m} \frac{j_{n+m}(x)}{x^{n+m}} .
$$

In fact, we superpose transverse electric and transverse magnetic fields in Eqs. (24) and (25). For convenience, we choose the normalisation coefficients $a$ and $b$ in Eq. (23a) inside the crystal so that they transform as $a=b$ before the crystal. Since we do not solve the boundary problem, such normalisation is justified.

In this assumption, the wave field before the crystal has only the right-hand polarised (RHP) component, so that $E_{1,-}(z=0$, before the crystal $)=0$. In the boundary plane $z=0$ the transverse electric field $\mathbf{E}_{1}^{(o)}$ before the crystal is converted into the same transverse electric field. The transverse magnetic field $E_{1}^{(e)}$ is converted in the same manner, though
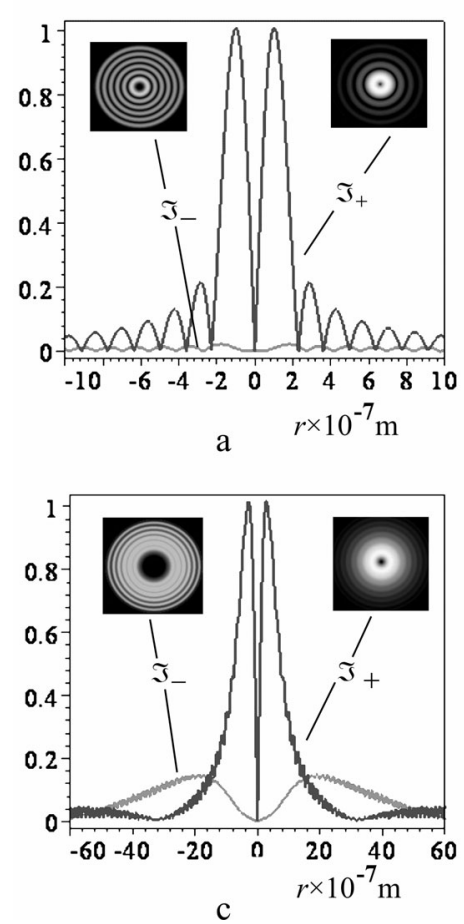
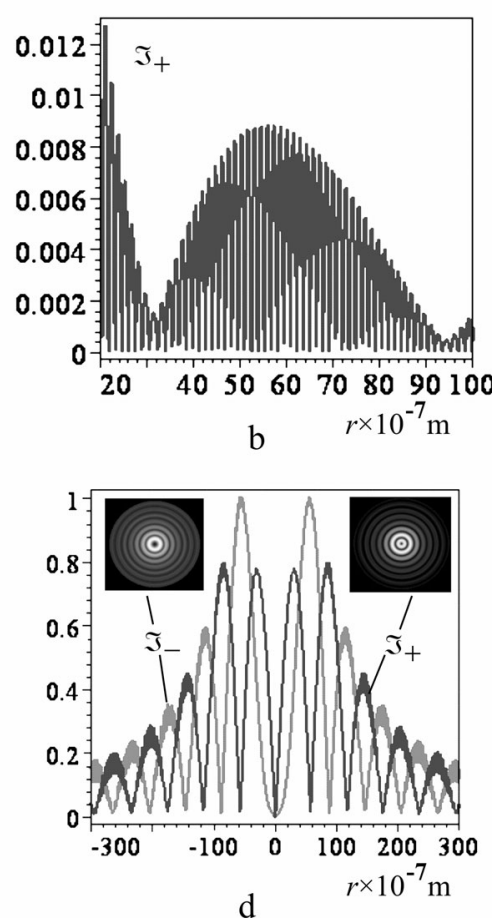

d

with the refractive index $n_{3}$ and the other scale for the $z$ coordinate. Naturally, in the $z=0$ plane inside the crys tal, the $E_{1,-}(z=0$, inside the crystal)

field component is nonzero over all the $z=0$ plane. Evanescent waves in both the ordinary and extraordinary beams do not permit the boundary field in the LHP component to be compensated. The field distribution for this case is shown in Fig. 1a. Since the contribution of evanescent waves is small, the ratio of 
total intensities for the LHP and RHP components is about 0.01 . As the crystal length increases, field oscillations are smoothed. However, a ripple seen in Fig. 1 on the envelope curves for the nonparaxial beam inside the crystal testifies upon fast oscillations in the beam at the initial plane. Evolution of the intensity distribution and the wave front shape for the beam components near the initial plane are shown in Fig. 2.
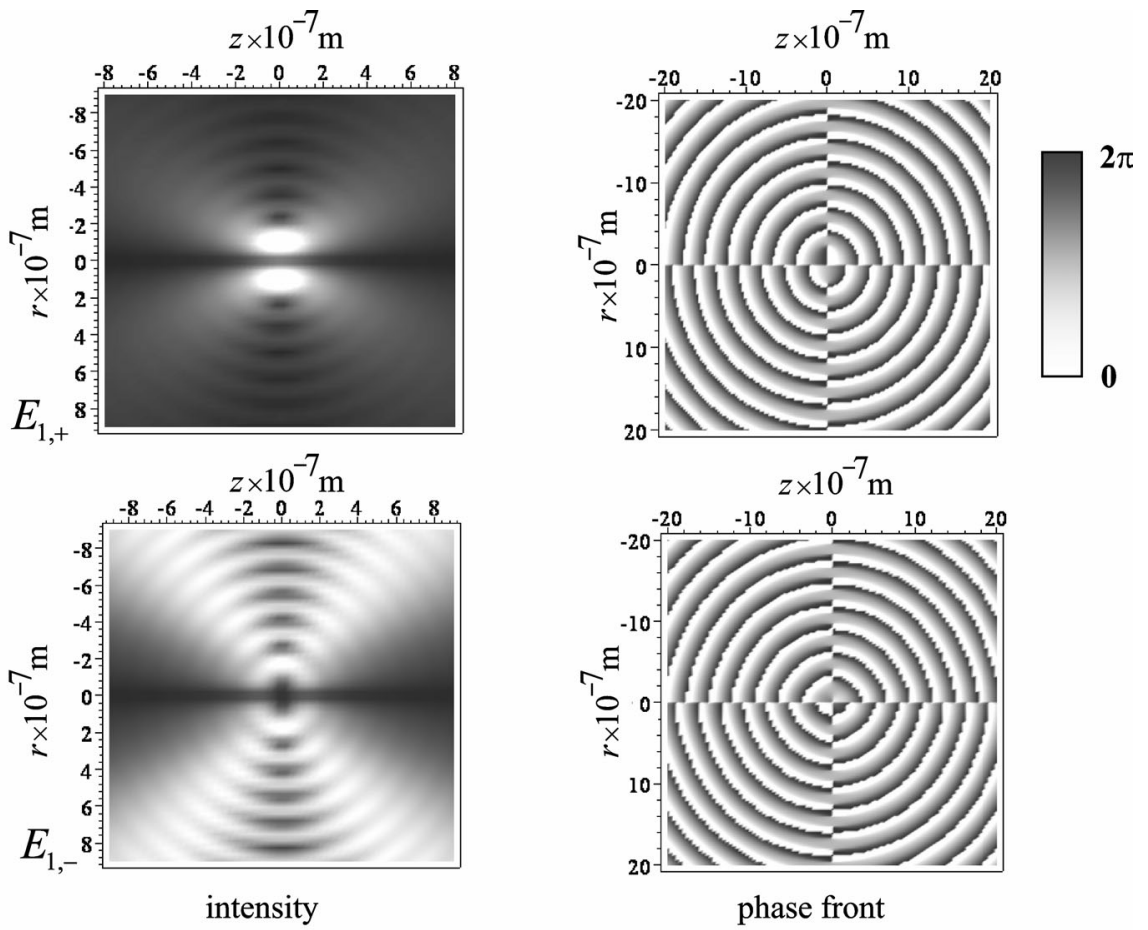

Fig. 2. Section in $\mathrm{r} z$ plane of intensity distribution and phase front for the circularly polarised components of nonparaxial beam with $w_{0}=1 \mu \mathrm{m}, l=-1$.

Let us now consider a paraxial approximation of the above field. Obviously, the factor in the brackets in $E_{1,-}$ is zero at $u v=r^{2}=0, z=0$. The complex radius $R_{o}$ in the vicinity of $r \approx 0$ may be found as

$$
\begin{aligned}
& k_{o} R_{o}=k_{o} \sqrt{u v+\left(z+i z_{o}\right)^{2}} \\
& =k_{o}\left(z+i z_{o}\right) \sqrt{1+\frac{u v}{\left(z+i z_{o}\right)^{2}}} \approx k_{o}\left(z+i z_{o}\right)+\frac{k_{o} r^{2}}{2\left(z+i z_{o}\right)},
\end{aligned}
$$

where $u v=r^{2}=x^{2}+y^{2}$ and we have taken into account that

$$
\left|\frac{r^{2}}{\left(z+i z_{o}\right)^{2}}\right| \ll<\text {. }
$$

However, the requirement given by Eq. (28) is nothing but the paraxial approximation for the wave beams, and 


$$
j_{1}(x)=\frac{\sin x}{x^{2}}-\frac{\cos x}{x} .
$$

In our case, we make use of the relation $\cos x=\left(e^{i x}+e^{-i x}\right) / 2$, so that

$$
\begin{aligned}
& \exp \left(-i k_{o} R_{o}\right) \approx \exp \left(-i k_{o} z\right) \exp \left(k_{o} z_{o}\right) \exp \left(-i k_{o} \frac{r^{2}}{2\left(z+i z_{o}\right)}\right), \\
& \approx \exp \left(-i k_{o} z\right) \exp \left(k_{o} z_{o}\right) \exp \left(-\frac{r^{2}}{w_{0}^{2} \sigma_{o}}\right)
\end{aligned}
$$

with $w_{0}=\sqrt{2 z_{o} / k_{o}}$ being the beam waist at the $z=0$ plane, $\sigma_{o}=1-i z / z_{o}$, and $\left|\exp \left(-i k_{o} R_{o}\right)\right| \gg>\left|\exp \left(i k_{o} R_{o}\right)\right|$. We obtain finally

$$
\cos \left(k_{o} R_{o}\right) \approx \exp \left(-i k_{o} z\right) \frac{\exp \left(k_{o} z_{o}\right)}{2} \exp \left(-\frac{r^{2}}{w_{0}^{2} \sigma_{o}}\right) .
$$

Let us also use the approximation $k_{o} R_{o} \approx k_{o}\left(z+i z_{o}\right)$ in the denominator of Eq. (29). Assuming that $\left|k_{o}\left(z+i z_{o}\right)^{2}\right|^{-1}<<\left|\left(z+i z_{o}\right)\right|^{-1}$ in Eq. (29), we come to the expression

$$
j_{1}\left(k_{o} R_{o}\right) \approx-\frac{\exp \left(k_{o} z_{o}\right)}{2} \frac{\exp \left(-i k_{o} z\right)}{k_{o}\left(z+i z_{o}\right)} \exp \left(-\frac{r^{2}}{w_{0}^{2} \sigma_{o}}\right) .
$$

For the extraordinary beam we have

$$
\cos \left(k_{e} R_{e}\right) \approx \exp \left(-i k_{e} z^{\prime}\right) \frac{\exp \left(k_{e} z_{e}\right)}{2} \exp \left(-\frac{r^{2}}{w_{0}^{2} \sigma_{e}}\right) .
$$

At the same time, using the same relations and Eq. (9), we obtain the relation $k_{e} z^{\prime}=k_{0} \sqrt{\varepsilon_{3}} z \sqrt{\varepsilon / \varepsilon_{3}}=k_{0} \sqrt{\varepsilon} z=k_{o} z$, implying that both the ordinary and the extraordinary beams propagate with the same phase velocities along the $z$ axis. Also, since we have $\quad \sigma_{e}=1-i \frac{z^{\prime}}{z_{e}}=1-i \sqrt{\varepsilon / \varepsilon_{3}} z /\left(k_{0} \sqrt{\varepsilon_{3}} w_{0}^{2} / 2\right)=1-i \frac{z}{z_{e}^{\prime}} \quad\left(\right.$ with $\quad z_{e}^{\prime}=k_{e}^{\prime} w_{0}^{2} / 2 \quad$ and $\left.k_{e}^{\prime}=\varepsilon_{3} / \sqrt{\varepsilon} k_{0}=n_{3}^{2} / n_{o} k_{0}\right)$, the complex amplitude of the paraxial extraordinary beam is characterised by its own wave number $k_{e}^{\prime}=n_{3}^{2} / n_{o}^{2} k_{o}$, contrary to the situation with the ordinary paraxial beam (the wave number $k_{o}$ ). Note also that

$$
j_{0}\left(k_{o} R_{o}\right) \approx i \exp \left(-i k_{o} z^{\prime}\right) \frac{\exp \left(k_{o} z_{o}\right)}{2 k_{o}\left(z+i z_{o}\right)} \exp \left(-\frac{r^{2}}{w_{0}^{2} \sigma_{o}}\right) \approx i j_{1}\left(k_{o} R_{o}\right) .
$$

Thus, we arrive at the transverse electric field for the paraxial vortex beams of the lowest order in the following form:

$$
E_{1,+} \sim \frac{v}{w_{0}}\left(\frac{G_{o}}{\sigma_{o}}+\frac{G_{e}}{\sigma_{e}}\right) e^{-i k_{o} z}, E_{1,-} \sim \frac{u}{w_{0}}\left(\frac{G_{o}}{\sigma_{o}}-\frac{G_{e}}{\sigma_{e}}\right) e^{-i k_{o} z},
$$


where $G_{o, e}=\exp \left(-r^{2} / w_{0}^{2} \sigma_{o, e}\right) / \sigma_{o, e}$.

Evolution of the beam profiles shown in Fig. 3 demonstrates gradual transition of the beam state from the nonparaxial to paraxial region of the beam's parameters.

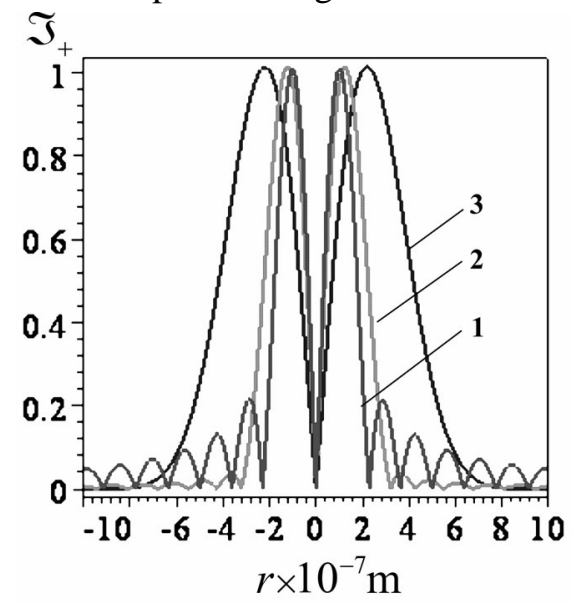

Fig. 3. Radial intensity distribution $\mathfrak{I}_{+}$in the RHP component for different beam waists $w_{0}$ in the $z=0$ plane: curve $1-w_{0}=1.0 \mu \mathrm{m}$, curve $2-w_{0}=1.5 \mu \mathrm{m}$, and curve $3-w_{0}=3.0 \mu \mathrm{m}$.

\subsection{Nonparaxial Gaussian beam}

\subsubsection{Circular polarisation}

Generally speaking, wave fields with a smooth envelope lacking phase singularities do not represent mode beams with eigen polarisations, in contrast to the vortex-bearing beams which can be obtained by simple differentiation over $u$ and $v$ variables, as made in the previous section. Nevertheless, these fields may be found by means of integral operators. Indeed, let us integrate Eqs. (16) over the $u$ variable:

$$
\begin{aligned}
& E_{0,+}^{o}=\int \partial_{u} \Psi_{1} d u=\Psi_{1}, \\
& E_{0,-}^{o}=-\int \partial_{v} \Psi_{1} d u .
\end{aligned}
$$

Choosing the generatrix function $\Psi_{1}$ in the form of Eq. (13) and using Eq. (32) for the paraxial approximation, we come to the Gaussian envelope for the RHP component:

$$
E_{0,+}^{o}\left(x, y, z, k_{o}\right)=\frac{j_{0}\left(k_{o} R_{o}\right)}{j_{0}\left(i k_{o} z_{o}\right)} .
$$

With the aid of Eq. (26) we obtain

$$
E_{0,-}^{o}=-\frac{u}{v} j_{0}\left(k_{o} R_{o}\right)+\frac{1}{v} \int j_{o}\left(k_{o} R_{o}\right) d u
$$

for the LHP component. Taking into account Eq. (15), we find

$$
E_{0,-}^{o}=-\left\{\frac{u}{v} j_{0}\left(k_{o} R_{o}\right)-\frac{2}{k_{o}^{2} v^{2}} \cos \left(k_{o} R_{o}\right)\right\} / j_{0}\left(i k_{o} z_{o}\right) \text {. }
$$


Similarly we obtain the partial extraordinary field:

$$
\begin{gathered}
E_{0,+}^{e}\left(x, y, z^{\prime}, k_{e}\right)=\frac{j_{0}\left(k_{e} R_{e}\right)}{j_{0}\left(i k_{e} z_{e}\right)}, \\
E_{0,-}^{e}\left(x, y, z^{\prime}, k_{e}\right)=\left\{\frac{u}{v} j_{0}\left(k_{e} R_{e}\right)-\frac{2}{k_{e}^{2} v^{2}} \cos \left(k_{e} R_{e}\right)\right\} / j_{0}\left(i k_{e} z_{e}\right) .
\end{gathered}
$$

However, the above partial ordinary and extraordinary beams cannot exist by themselves because they have amplitude singularities at the $r=0$ axis. The partial beams produce a stable superposition $\mathbf{E}_{0}=\mathbf{E}_{0}^{o}+\mathbf{E}_{0}^{e}$ lacking amplitude singularities:

$$
\begin{gathered}
E_{0,+}(x, y, z)=\frac{j_{0}\left(k_{o} R_{o}\right)}{j_{0}\left(i k_{o} z_{o}\right)}+\frac{j_{0}\left(k_{e} R_{e}\right)}{j_{0}\left(i k_{e} z_{e}\right)}, \\
E_{0,-}(x, y, z)=-\left\{\frac{u}{v}\left[\frac{j_{0}\left(k_{o} R_{o}\right)}{j_{0}\left(i k_{o} z_{o}\right)}-\frac{j_{0}\left(k_{e} R_{e}\right)}{j_{0}\left(i k_{e} z_{e}\right)}\right]-\frac{2}{v^{2}}\left[\frac{\cos \left(k_{o} R_{o}\right)}{k_{o}^{2} j_{0}\left(i k_{o} z_{o}\right)}-\frac{\cos \left(k_{e} R_{e}\right)}{k_{e}^{2} j_{0}\left(i k_{e} z_{e}\right)}\right]\right\} .
\end{gathered}
$$

The expressions obtained above transform into typical paraxial ones under the paraxial condition given by Eq. (28):

$$
\begin{aligned}
& E_{0,+}(x, y, z) \sim\left(G_{o}+G_{e}\right) e^{-i k_{o} z}, \\
& E_{0,-} \sim-\left\{\frac{u}{v}\left[G_{o}-G_{e}\right]+\frac{w_{0}^{2}}{v^{2}}\left[\sigma_{o} G_{o}-\sigma_{e} G_{e}\right]\right\} e^{-i k_{o} z} .
\end{aligned}
$$

The field thus obtained describes propagation of the beam with the Gaussian envelope in the initial plane $z=0$. The RHP component has also the Gaussian envelope in each partial beam, whereas the LHP component has more complex shape of the envelope, bearing a doubly charged centred optical vortex.

\subsubsection{Linear polarisation}

A treatment of nonparaxial Gaussian beam with the initial linear polarisation needs a more detailed consideration of the structure of nonparaxial field. Any linearly polarised field may be presented as a sum of two circularly polarised ones, with the same field distributions in the RHP and LHP components at the crystal input. The beam with the initial field distribution like that given by Eqs. (30) and (31) may be formed when integration in Eq. (34) is performed over the $v$ variable rather than $u$ one. Then the field components may be written as

$$
\begin{aligned}
\bar{E}_{0,-}\left(x, y, z^{\prime}\right) & =-\left\{\frac{v}{u}\left[\frac{j_{0}\left(k_{o} R_{o}\right)}{j_{0}\left(i k_{o} z_{o}\right)}-\frac{j_{0}\left(k_{e} R_{e}\right)}{j_{0}\left(i k_{e} z_{e}\right)}\right]-\frac{2}{u^{2}}\left[\frac{\cos \left(k_{o} R_{o}\right)}{k_{o}^{2} j_{0}\left(i k_{o} z_{o}\right)}-\frac{\cos \left(k_{e} R_{e}\right)}{k_{e}^{2} j_{0}\left(i k_{e} z_{e}\right)}\right]\right\}, \\
\bar{E}_{0,-}(x, y, z) & =\left[\frac{j_{0}\left(k_{o} R_{o}\right)}{j_{0}\left(i k_{o} z_{o}\right)}+\frac{j_{0}\left(k_{e} R_{e}\right)}{j_{0}\left(i k_{e} z_{e}\right)}\right] .
\end{aligned}
$$



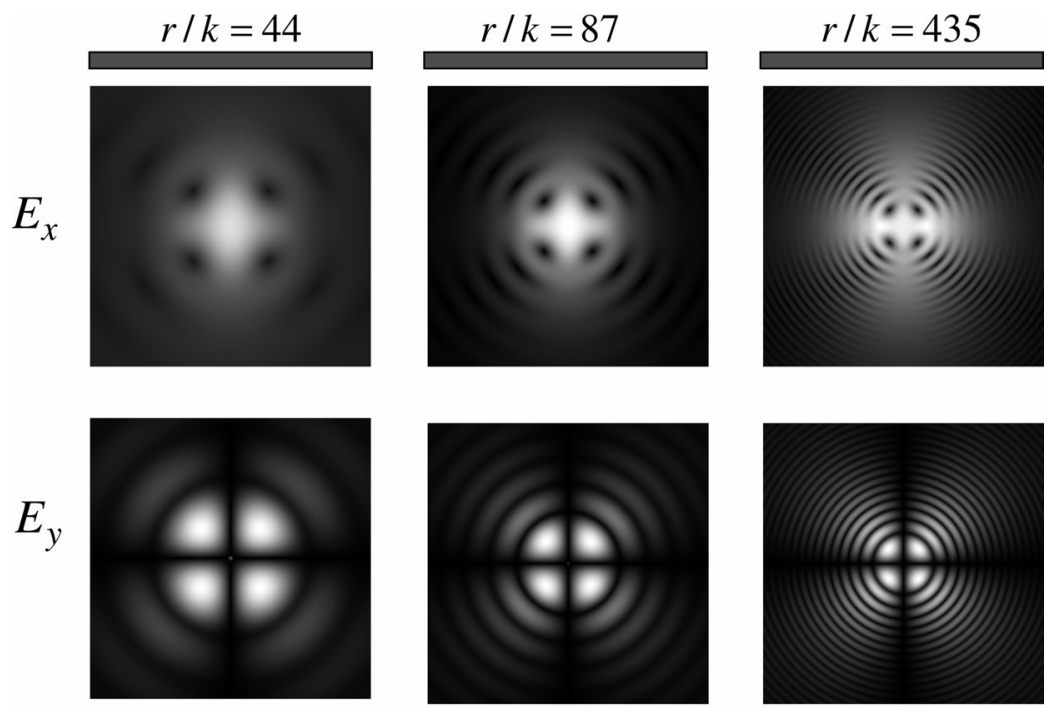

$z / k=200$

$z / k=40$

$z / k=200$

Fig. 4. Evolution of conoscopic pattern for the $E_{x}$ and $E_{y}$ components of nonparaxial beam with the initial linear polarization directed along $x$ and the waist radius $w_{0} / k=1$, which is observed along the crystal with $n_{o} / n_{3}=1.1$.

The components of the beam with the initial linear polarisation are $E_{+}^{(L)}=E_{+}+\bar{E}_{+}$ and $E_{-}^{(L)}=E_{-}+\bar{E}_{-}$. This beam composition includes the beams with different vortices ( $l=0, l=2$ and $l=-2$ ). Besides, the scales for the partial beams along the $z$ axis are different. As a result, the projection of linearly polarised electric field onto the cross section of the beam has also different scales along the $x$ and $y$ axes, unlike the circularly polarised beams. The pattern observed must be elliptically deformed. Fig. 4 illustrates the evolution of the conoscopic patterns for the field components $E_{x}$ and $E_{y}$ along the crystal length, provided that the initial linearly polarised field at the crystal input has the circular symmetry. As the nonparaxial beam propagates along the crystal, a contour of the conoscopic pattern shows through a dark background. Although the Maltese cross for the $E_{y}$ component has a standard shape, the pattern for the $E_{x}$ component is slightly deformed even at a small propagation distance comparable with the light wavelength. The vortex positions in a set of topological quadruples forming "the white cross", have different coordinates along the $x$ and $y$ axes. Deformation of the conoscopic pattern for the $E_{x}$ component increases when the beam propagates further on. The elliptical deformation is nonuniformly distributed through the cross section of the beam. It grows quickly as the observation point moves away from the optic axis, vanishing in practice nearby this axis. However, the elliptical deformation cannot exceed the value $a / b=n_{o} / n_{3}$, with $a$ and $b$ being the axes of the intensity ellipse. Such a deformation effect represents geometrical manifestation of the interference between nonparaxial wave beams (ordinary and extraordinary) having different spatial scales. 
Notice that deformation of the pattern does not vanish even in the paraxial case [14, 18 , 24], being inherent to linearly polarised beams. The above results are consistent with the theoretical analysis $[18,25,26]$ for paraxial beams propagating perpendicular or nearly perpendicular to the optic axis in crystals. At the same time, this deformation is very difficult to detect experimentally for the beams propagating along the optic axis of any real crystals, since it is proportional to the ratio of refractive indices for the ordinary and extraordinary beams. The effect is comparable with an astigmatic aberration imposed by lens systems in nonparaxial beams. Nevertheless, the elliptical deformation has been experimentally observed when the beam propagates nearly perpendicular to the optic axis [25]. In the latter case rotation of the crystal would entail a precession of the beam axis.

\subsection{High-order nonparaxial vortex beams}

High-order wave beams bearing optical vortices and ring dislocations in a uniaxial crystal, which have standard analogues in the paraxial approximation, may be produced by means of the following procedure:

$$
\mathbf{E}_{m}^{|n-m|,(o, e)}=\left(\begin{array}{c}
\partial_{u}^{m+1} \partial_{v}^{n} \\
\mp \partial_{v}^{n+1} \partial_{u}^{m}
\end{array}\right) j_{0}\left(k_{o, e} R_{o, e}\right),
$$

with the signs $(\mp)$ being associated with the ordinary and extraordinary beams, respectively. Indeed, at first we will assume that $n \geq m$ and find the function

$$
\frac{\partial^{n}}{\partial v^{n}} j_{0}\left(k_{o, e} R_{o, e}\right)=(-1)^{n}\left(\frac{k_{o, e}}{2}\right)^{n}\left(\frac{u}{R_{o, e}}\right)^{n} j_{n}\left(k_{o, e} R_{o, e}\right),
$$

where we have used Eq. (26). It is seen from Eq. (44) that the operator $\partial_{v}$ corresponds to an event of birth of the vortex with a positive topological charge, whereas the operator $\partial_{v}^{n}$ begets the $n$-charged optical vortex. Similarly, the operator $\partial_{u}$ corresponds to an event of birth of the vortex with a negative topological charge, whereas the operator $\partial_{u}^{m}$ gives rise to the $m$-charged optical vortex. At the same time, the operator $\partial_{v}^{m} \partial_{u}^{m}$ is topologically neutral, being responsible for appearance of ring dislocations in the beam, at least in the $z=0$ plane.

The common action of the operators $\partial_{u}^{n} \partial_{v}^{m}$ manifests itself as follows:

$$
\begin{aligned}
& \frac{\partial^{n}}{\partial v^{n}} \frac{\partial^{m}}{\partial u^{m}} j_{0}\left(k_{o, e} R_{o, e}\right)=(-1)^{n}\left(\frac{k_{o, e}}{2}\right)^{n} \frac{\partial^{m}}{\partial u^{m}}\left[u^{n}\left(\frac{j_{n}\left(k_{o, e} R_{o, e}\right)}{R_{o, e}}\right)\right] \\
& =(-1)^{n}\left(\frac{k_{o, e}}{2}\right)^{n} \sum_{j=0}^{m}\left(\begin{array}{c}
m \\
m-j
\end{array}\right) \frac{\partial^{m}}{\partial u^{m}}\left[\frac{\partial^{m-j}}{\partial u^{m-j}} u^{n} \frac{\partial^{j}}{\partial u^{j}} \frac{j_{n}\left(k_{o, e} R_{o, e}\right)}{R_{o, e}}\right] \\
& =(i)^{n}\left(\frac{1}{w_{0}^{2}}\right)^{n}\left(\frac{u}{\zeta_{o, e}}\right)^{n-m}\left(\frac{1}{\zeta_{o, e}}\right)^{m} \sum_{j=0}^{m}\left(\begin{array}{c}
m \\
m-j
\end{array}\right) a_{j}\left(\frac{k_{o, e} r^{2}}{2 R_{o, e}}\right)^{j} j_{n+j}\left(k_{o, e} R_{o, e}\right),
\end{aligned}
$$

Ukr. J. Phys. Opt. 2009, V11, №1 
where $a_{j}=n(n-1)(n-2) \ldots(n-m+j-1) \quad$ and $\quad$ we have employed the rules $\partial_{x}^{m}[f \cdot \varphi]=\sum_{j=0}^{m}\left(\begin{array}{c}m \\ j\end{array}\right) \partial_{x}^{j} f \partial_{x}^{m-j} \varphi$ and $\zeta_{o, e}=\sqrt{1+\frac{u v}{\left(z+i z_{o, e}\right)^{2}}}$. Thus, we obtain a wave beam bearing a centred optical vortex with the $(n-m)$-topological charge, while a radial dependence of the wave beam is defined by a complex function

$$
Q_{n}^{n-m,(o, e)}(r)=\left(\frac{1}{\zeta_{o, e}}\right)^{m} \sum_{j=0}^{m}\left(\begin{array}{c}
m \\
m-j
\end{array}\right) a_{j}\left(\frac{k_{o, e} r^{2}}{2 R_{o, e}}\right)^{j} j_{n+j}\left(k_{o, e} R_{o, e}\right) .
$$

Let us derive some simple rules for transition from the nonparaxial to paraxial beams with the Gaussian envelope. A recurrence relation for the spherical Bessel functions is given by $j_{n-1}(x)+j_{n+1}(x)=\frac{2 n+1}{x} j_{n}(x)$. Then, taking into account the paraxial requirement given by Eq. (28), we come to the relations for the paraxial transition [10]:

$$
\begin{aligned}
& j_{2 p}\left(k_{o, e} R_{o, e}\right) \rightarrow-j_{2 p-2}\left(k_{o, e} R_{o, e}\right), \\
& j_{2 p+1}\left(k_{o, e} R_{o, e}\right) \rightarrow-j_{2 p-1}\left(k_{o, e} R_{o, e}\right),
\end{aligned}
$$

as well as complimentary relations obtained from Eq. (32):

$$
\begin{aligned}
& j_{1}\left(k_{o, e} R_{o, e}\right) \rightarrow i j_{o}\left(k_{o, e} R_{o, e}\right), \\
& j_{2}\left(k_{o, e} R_{o, e}\right) \rightarrow-j_{o}\left(k_{o, e} R_{o, e}\right), \\
& \frac{1}{R_{o, e}}=\frac{1}{i z_{o, e} \zeta_{o, e}} \rightarrow \frac{1}{i z_{o, e} \sigma_{o, e}} .
\end{aligned}
$$

Finally, the radial function $Q(r)$ in Eq. (46) may be transformed to a standard expression:

$$
Q_{n}^{n-m,(o, e)}(r) \rightarrow(-1)^{m+n} n ! \frac{(i)^{n}}{\sigma^{n}} L_{n}^{(n-m)}\left(\frac{r^{2}}{w_{0}^{2} \sigma_{o, e}}\right) G_{o, e},
$$

where we have made use of definition of the generalised Laguerre polynomial:

$$
L_{n}^{\lambda}(x)=\sum_{j=0}^{n} \frac{(-1)^{j}}{j !}\left(\begin{array}{c}
n+\lambda \\
n-j
\end{array}\right) x^{j} .
$$

Thus, Eqs. (43) finally normalised for the high-order nonparaxial beams under the condition $n>m$ may be rewritten as

$$
\begin{aligned}
& E_{n,+}^{n-m}=\left(\frac{u}{\zeta_{o}}\right)^{n-m-1} \frac{Q_{n}^{n-m-1,(o)}(r)}{j_{0}\left(i k_{o} z_{o}\right)}+\left(\frac{u}{\zeta_{e}}\right)^{n-m-1} \frac{Q_{n}^{n-m-1,(e)}(r)}{j_{0}\left(i k_{e} z_{e}\right)}, \\
& E_{n,-}^{n-m}=\left(\frac{u}{\zeta_{o}}\right)^{n-m+1} \frac{Q_{n+1}^{n+1-m,(o)}(r)}{j_{0}\left(i k_{o} z_{o}\right)}-\left(\frac{u}{\zeta_{e}}\right)^{n-m+1} \frac{Q_{n+1}^{n+1-m,(e)}(r)}{j_{0}\left(i k_{e} z_{e}\right)},
\end{aligned}
$$


whereas for the case of $n<m$ we have

$$
\begin{aligned}
& E_{m,+}^{m-n}=\left(\frac{v}{\zeta_{o}}\right)^{m-n-1} \frac{Q_{m}^{m-n-1,(o)}(r)}{j_{0}\left(i k_{o} z_{o}\right)}+\left(\frac{v}{\zeta_{e}}\right)^{m-n-1} \frac{Q_{m+1}^{m-n-1,(e)}(r)}{j_{0}\left(i k_{e} z_{e}\right)} \\
& E_{m,-}^{m-n}=\left(\frac{v}{\zeta_{o}}\right)^{m-n+1} \frac{Q_{m+1}^{m-n+1,(o)}(r)}{j_{0}\left(i k_{o} z_{o}\right)}-\left(\frac{v}{\zeta_{e}}\right)^{m-n-1} \frac{Q_{m}^{m-n-1,(e)}(r)}{j_{0}\left(i k_{e} z_{e}\right)} .
\end{aligned}
$$

If $n>m$, the beam carries over a centred positively charged vortex with the topological charge $(n-m-1)$ and $n$-ring dislocations (toroidal vortices in the $z=0$ plane) in the RHP component, whereas the LHP component carries over a positively charged vortex with the topological charge $(n-m+1)$ and $n+1$-ring dislocations (toroidal vortices in the $z=0$ plane). If $m>n$, the beam carries over a negatively charged vortex with the topological charge $(m-n-1)$ and $m$-ring dislocations (toroidal vortices in the $z=0$ plane) in the RHP component and the LHP component carries over a negatively charged vortex with the topological charge $(m-n+1)$ and $m+1-$ ring dislocations (toroidal vortices in the $z=0$ plane).
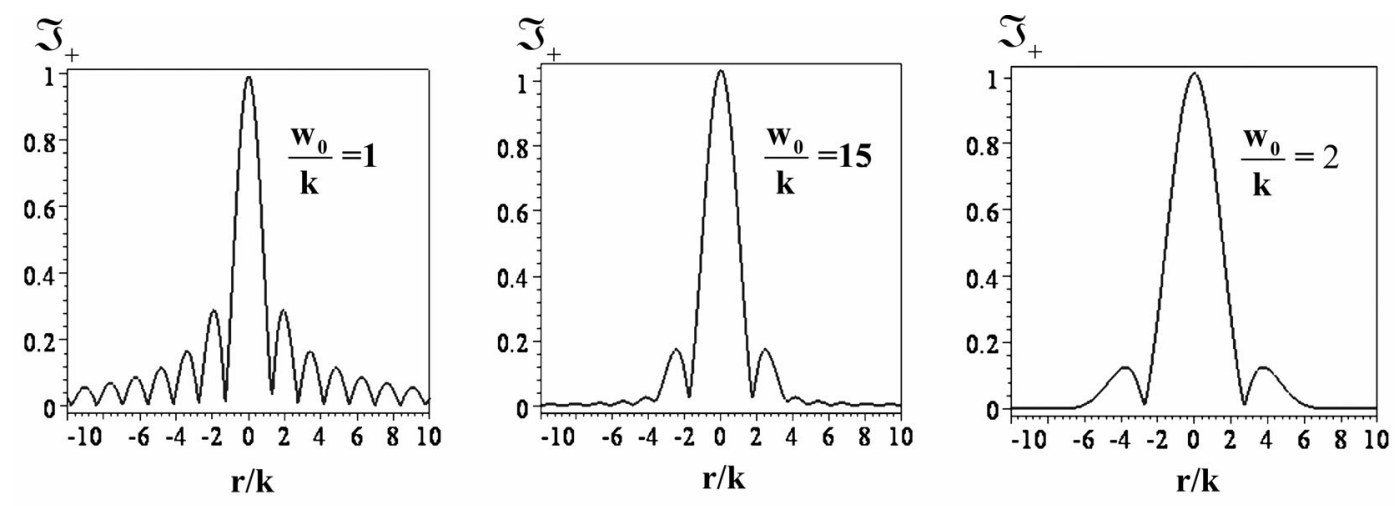

Fig. 5. Transformation of nonparaxial beam with $m=1$ and $I=0$ into a standard paraxial Laguerre-Gaussian beam under the condition of increasing beam waist $w_{0}$.

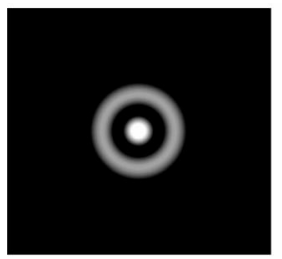

$\mathrm{w}_{0} / \mathrm{k}=\mathbf{1}$

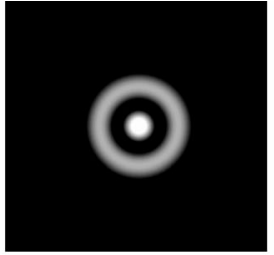

$\mathrm{w}_{0} / \mathrm{k}=1.2$

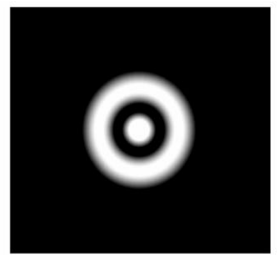

$\mathrm{w}_{0} / \mathrm{k}=1.5$

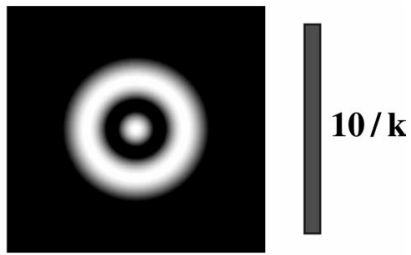

$\mathbf{w}_{0} / \mathbf{k}=\mathbf{2}$

Fig. 6. Transformation of intrinsic annular structure of the intensity for the RHP component of nonparaxial beam with $I=0$ and $m=2$ into a standard Laguerre-Gaussian beam under the condition of increasing beam waist $w_{0}$ at the crystal input $z=0$. 


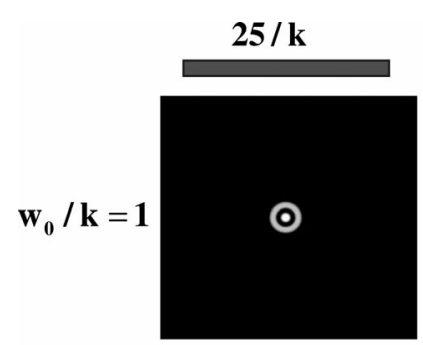

$\mathbf{z} / \mathbf{k}=0$

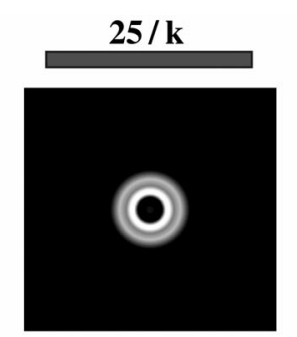

1.5

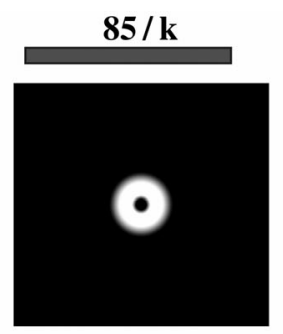

5

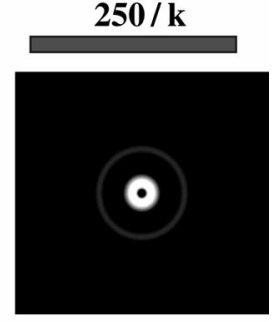

10

Fig. 7. Conversion of intrinsic annular structure of the RHP component of nonparaxial beam with $I=0$ and $m=2$ into a typical conoscopic pattern along the crystal length.

Fig. 5 and Fig. 6 illustrate transformations of the beam profiles for the case of low orders $l=m-n-1=0, n=1$ and $l=0, n=2$ and the nonparaxial beam into those typical for the paraxial Laguerre-Gaussian beam. Notice that the lateral oscillations of the beam intensity become nearly completely smoothed for the beam waist about $w_{0} \sim 2 \lambda$, when the influence of evanescent waves even on higher-order nonparaxial beam profile is essentially weakened. When the nonparaxial beam of the highest order propagates along the crystal, an intrinsic annular pattern vanishes, being replaced by the typical conoscopic pattern. Fig. 7 shows a gradual transition of the annular picture into the conoscopic pattern peculiar for divergent light in a uniaxial crystal.

\section{Conclusions}

We have treated the propagation of nonparaxial beams along the optic axis of a uniaxial crystal. We have chosen a shape of generatrix beam in such a way that it have a standard form of Laguerre-Gaussian beam in the paraxial approximation. We have revealed that the behaviour of nonparaxial beams may be described in the framework of complexsource-point technique. The above method leads to nonparaxial beams with the eigen polarisations. This means that this mode beam propagates along the crystal without structural transformations of its field up to a scale factor.

There are two types of those mode beams, the transverse electric (TE) and transverse magnetic (TM) fields, that propagate in the crystal with different wave parameters. The mode beams have different scales along the $z$ axis. A beam with arbitrary field distribution at the crystal input is described as a superposition of the beams with eigen polarisations. These types of nonparaxial vortex beams have a circular symmetry through the beam cross section, being smoothly converted into their paraxial analogues. The centred optical vortices embedded in the beams do not lose their structural stability when either propagating or being focused tightly. The circular symmetry in the field distribution gets lost when the beam waist of the linearly polarised field becomes comparable with the light wavelength. Its cross section is deformed, i.e. the scales along the $x$ and $y$ axes become different. The beam deformation diminishes, though does not vanish completely, for the paraxial linearly polarised beams. 


\section{Acknowledgment}

The author thanks A. V. Volyar for his fruitful consideration of the theoretical results.

\section{References}

1. Soskin M and Vasnetsov M, 2001. Singular optics. Progr. Opt. 42: 219-276.

2. Volyar A and Fadeyeva T, 2003. Generation of singular beams in uniaxial crystals, Opt. Spectrosc. 94: 264-274.

3. Volyar A and Fadeyeva T, 2003. Destruction and syntheses of polarization umbilics in singular beams in crystals. Opt. Spectrosc. 95: 285-293.

4. Sheppard C and Saghafi S, 1998. Beam modes beyond the paraxial approximation: A scalar treatment. Phys. Rev. A. 57: 2971-2979.

5. Pattanayak D and Agraval G, 1980. Representation of vector electromagnetic beams. Phys. Rev. A. 22: 1159-1163.

6. Sheppard C and Saghafi S, 1999. Electric and magnetic dipole modes beyond the paraxial approximation. Optik. 110: 487-491.

7. Couture $\mathrm{M}$ and Belanger P, 1981. From Gaussian beam to complex-source-point wave. Phys. Rev. A. 24: 355-359.

8. Volyar A, Shvedov V and Fadeyeva T, 2001. Structure of nonparaxial Gaussian beams near the focus: 2. Optical vortices. Opt. Spectrosc. 90: 104-112.

9. Volyar A, Shvedov V and Fadeyeva T, 2001. Structure of nonparaxial Gaussian beams near the focus: 3. Stability, eigen modes and vortices. Opt. Spectrosc. 91: 255266.

10. Volyar A and Fadeyeva T, 2002. Dynamics of topological multipoles: High-order nonparaxial singular beams. Opt. Spectrosc. 92: 243-252.

11. Born M and Wolf E, Principles of optics. New York: Cambridge University Press (1999).

12. Ciattoni A, Cincotti G and Palma C, 2003. Circular polarized beams and vortex generation in uniaxial media. J. Opt. Soc. Amer. A. 20: 163-171.

13. Cincotti G, Ciattoni A and Sapia C, 2003. Radially and azimuthally polarized vortices in uniaxial crystals. Opt. Commun.220: 33-40.

14. Cincotti G, Ciatoni A and Palma C, 2002. Laguerre-Gaussian and Bessel-Gaussian beams in uniaxial crystals. J. Opt. Soc. Amer. A. 19: 1680-1688.

15. Berry M and Dennis M, 2003. The optical singularities of birefringent dichroic chiral crystals. Proc. Roy. Soc. Lond. A. 459: 1261-1292.

16. Berry M, 2004. Conical diffraction asymptotics: fine structure of Poggendorff rings and axial spike. J. Opt: Pure Appl. Opt. 6: 289-300.

17. Berry M, 2005. The optical singularities of bianisotropic crystals. Proc. Roy. Soc. A. 461: 2071-2098.

18. Seshadri S R, 2003. Basic elliptical Gaussian wave and beam in a uniaxial crystal. J. Opt. Soc. Amer. A. 20: 1818-1826.

19. Chin S Y and Felson L B, 1974. Gaussian beam in anisotropic media. Appl. Phys. 5: 225-239.

Ukr. J. Phys. Opt. 2009, V11, №1 
20. Fleck $\mathbf{J}$ A and Feit M D, 1983. Beam propagation in uniaxial anisotropic media. J. Opt. Soc. Amer. 73: 920-928.

21. Volyar A and Fadeyeva T, 2006. Laguerre-Gaussian beams with complex and real arguments in uniaxial crystals. Opt. Spectrosc. 101: 297-304.

22. Volyar A and Fadeyeva T, 2005. Laguerre-Gaussian beams in uniaxial crystals. Ukr. J. Phys. Opt. 5: 81-86.

23. Fadeyeva T, Rubass A, Egorov Yu, Volyar A and Swartzlander G, 2008. Quadrefringence of optical vortices in a uniaxial crystal. J. Opt. Soc. Amer. A. 25: 16341641

24. Fadeyeva T A, Rubass A F and Volyar A V, 2009. Transverse shift of a high-order paraxial vortex-beam induced by a homogeneous anisotropic medium. Phys. Rev. A. 79: 053815. 1-12.

25. Fadeyeva T A, Rubass A F, Sokolenko B V and Volyar A V, 2009. The vortex-beam 'precession' in a rotating uniaxial crystal. J. Opt. A: Pure Appl. Opt. 11: 094008.1-8.

26. Ciattoni A and Palma C, 2003. Optical propagation in uniaxial crystals orthogonal to the optic axis: paraxial theory and beyond. J. Opt. Soc. Amer. A. 20: 2163-2171.

Fadeyeva T., 2010. Nonparaxial wave beams in a uniaxial birefringent crystal. Ukr.J.Phys.Opt. 11: 44-60.

Анотація. Отримані розв'язки векторного хвильового рівняння для не параксіального променя, який поширюеться вздовж оптичної осі в одновісному двозаломлюючому кристалі. Виявилось, що циркулярно поляризований не параксіальний промінь може бути представлений, як набір поперечних електричних і магнітних хвиль, які при поширенні зберігають свою структуру з точністю до масштабного фактору. Промінь з довільним розподілом поля може бути записаний, як композиція таких хвильових полів. Показано, що циркулярносиметричний вихровий промінь з початково ииркулярною поляризаиією зберігає свою структуру в кристалі, тоді як ииркулярна симетрія не параксіального, вихрового променя з початково лінійною поляризацією втрачається при поширенні через кристал. Циркулярна симетрія не зберігається в параксіальному випадку, будучи природною властивістю лінійно-поляризованого променя. 